\title{
Multivisceral Resection for Colonic Splenic Flexure Malakoplakia: A Minimally Invasive Approach
}

\author{
Andrés Ramiro Lanza Díaz ${ }^{1}$, Santiago Gallardo Pezet ${ }^{1}$, Osvaldo Soto González ${ }^{2}$, Montserrat Guraieb Trueba ${ }^{1}$, \\ Ivan Azael Martínez Alonso ${ }^{3}$, Mario Alberto López Ramirez ${ }^{1}$ \\ ${ }^{1}$ Division of Colorectal Surgery, Surgery Department, Hospital Central Militar, Secretaría de la Defensa Nacional, Mexico City; ${ }^{2}$ Pathology \\ Department, Hospital Central Militar, Secretaría de la Defensa Nacional, Mexico City; ${ }^{3}$ Urology Department, Hospital Central Militar, \\ Secretaría de la Defensa Nacional, Mexico City, Mexico
}

\begin{abstract}
Malakoplakia is a rare granulomatous inflammatory disorder. Its diagnosis depends on histopathological findings; however, high-quality literature regarding proper medical/surgical treatment is lacking. A 38-year-old diabetic female patient was admitted to the emergency room with a history of lower gastrointestinal hemorrhage. Colonoscopy revealed a lesion in the descending colon, and abdominal computed tomography revealed a splenic flexure mass involving the lower pole of the spleen and upper pole of the left kidney. Biopsies confirmed the diagnosis of malakoplakia. After completing antibiotic treatment, a restaging computed tomography revealed a discrete mass increase; hence, the patient underwent laparoscopic en bloc colectomy and partial nephrectomy. Postoperatively, the patient developed a pancreatic fistula, which was successfully treated with percutaneous drainage and antibiotics. The presence of pathognomonic Michaelis-Gutmann inclusions on histopathology is frequently reported as the key to diagnosing malakoplakia. Herein, we present a successful, minimally invasive surgical treatment for colonic malakoplakia.
\end{abstract}

Keywords: Malakoplakia; Minimally invasive surgical procedures; Colectomy; Nephrectomy; Type 2 diabetes mellitus

\section{INTRODUCTION}

Malakoplakia is an extremely rare granulomatous inflammatory disorder. Its name is derived from the Greek words malakos (soft) and plakos (plate). Since 1965, 26 cases of colonic involvement have been reported to date. It was first described in 1901 by Hansemann, followed by Michaelis and Gutmann in 1902, and since then it has been considered histologically unique. Malakoplakia mainly involves the genitourinary tract, followed by the gastrointestinal tract, and can also affect the lung, brain, adrenal glands,

Received: Mar 11, 2021 - Revised: May 27, 2021 - Accepted: May 29, 2021 Correspondence to: Andrés Ramiro Lanza Díaz, M.D.

Tel: +52-55-64946182, Fax: +57-3214492317

Division of Colorectal Surgery, Surgery Department, Hospital Central Militar, Periférico Blvrd Manuel Ávila Camacho s/n, Militar, Miguel Hidalgo, 11200

Mexico City, Mexico

E-mail: andreslanzadiaz@gmail.com

ORCID: https://orcid.org/0000-0002-6430-9029

(C) 2021 The Korean Society of Coloproctology

This is an open-access article distributed under the terms of the Creative Commons Attribution NonCommercial License (https://creativecommons.org/licenses/by-nc/4.0) which permits unrestricted noncommercial use, distribution, and reproduction in any medium, provided the original work is properly cited. skin, pancreas, and less frequently the retroperitoneum, with the signs and symptoms being widely diverse [1].

Its etiology remains unknown, and although it has been associated with some degree of immunosuppression (human immunodeficiency virus [HIV], neoplasms, steroid use, diabetes mellitus $[D M]$, posttransplantation, etc.), it can also occur in immunocompetent patients [2]. The initial cause appears to be infectious, and the organisms responsible for this are believed to be Gramnegative bacilli, with Escherichia coli being the most frequently isolated organism. Regarding the pathophysiology, the most accepted hypothesis is an alteration in the process of bacterial phagocytosis due to a deficit in lysosomal functioning, and the characteristic and pathognomonic histopathological findings are the product of incomplete bacterial phagocytosis and the bacterial glycolipid residues that result in an accumulation of calcium and iron deposits, subsequently giving the lesion a granulomatous appearance [1]. An alteration in the functioning of T lymphocytes cannot be ruled out because of the most frequently affected population (HIV, transplanted patients, DM).

Herein, we present a clinical case of a young immunocompromised female patient who was successfully treated with minimally 


\section{Coloproctology $\quad$ Andrés Ramiro Lanza Diaz, et al.}

invasive multivisceral resection.

\section{CASE REPORT}

This study was approved by the Institutional Review Board of the Central Military Hospital (No. C.inv.-066). Written informed consent was obtained for publication of this case report and accompanying images.

A 38-year-old woman with a history of type $2 \mathrm{DM}$, who was insulin-dependent, and had poor glycemic control was admitted to the emergency room due to intense left flank pain and active hematochezia. She required multiple blood transfusions due to acute anemia (hemoglobin, $5.1 \mathrm{~g} / \mathrm{dL}$ ) and hemodynamic instability (blood pressure, $85 / 46 \mathrm{mmHg}$ ). On physical examination, left flank pain and tenderness were evident, and fresh blood was observed on digital rectal examination.
Colonoscopy revealed an elevated, subepithelial lesion in the descending colon, involving $50 \%$ of the circumference, with a decrease in the size of the colonic lumen, and no active bleeding. Biopsies were also performed (Fig. 1). The histopathological report showed unspecific granulation tissue, polymorphonuclear cells, and marked atrophic endothelial changes.

Abdominal computed tomography (CT) revealed a $7.3 \mathrm{~cm}$ lesion in the splenic flexure of the colon, with contact on the lower pole of the spleen and interface loss with suspected infiltration of the upper pole of the left kidney (Fig. 2). Given the CT findings, a percutaneous CT-guided biopsy was performed. Histopathological analysis showed von Hansemann cells (Fig. 3A), and Michaelis-Gutmann inclusions bodies were also identified (Fig. 3B). No malignant neoplastic cells were observed. Based on these findings, the patient was diagnosed with malakoplakia. A posteriorly oriented trajectory during endoscopic examination confirmed the
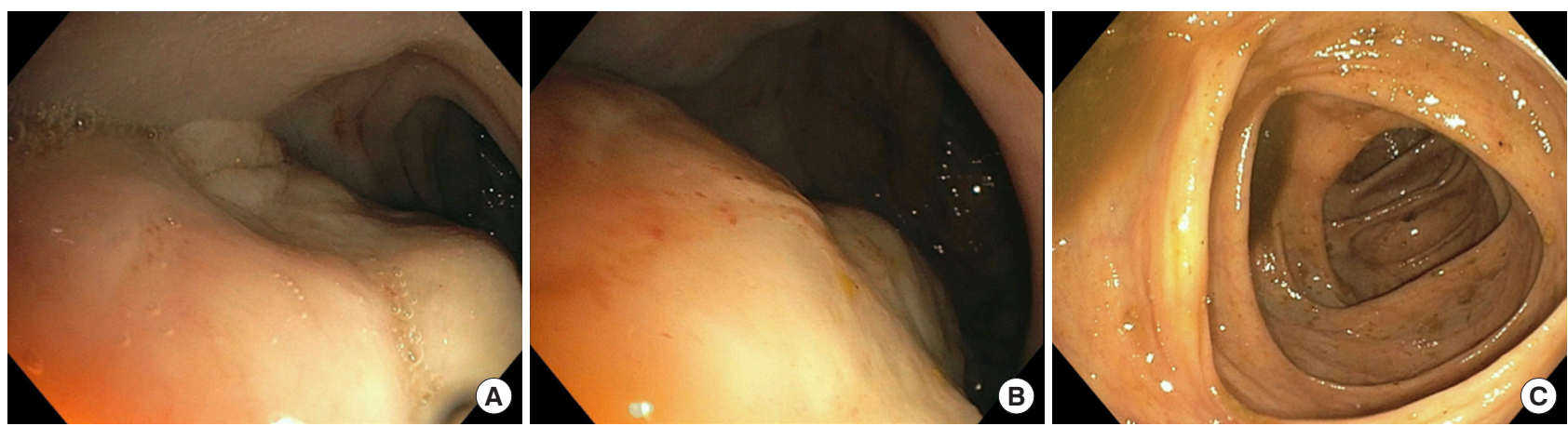

Fig. 1. Colonoscopy images. (A, B) Elevated extrinsic/submucosal lesion in the descending colon, approximately 33 to $38 \mathrm{~cm}$ from the anal margin, involving $50 \%$ of the circumference, without evidence of active or recent bleeding. (C) The transverse colon with an endoscopic normal appearance.
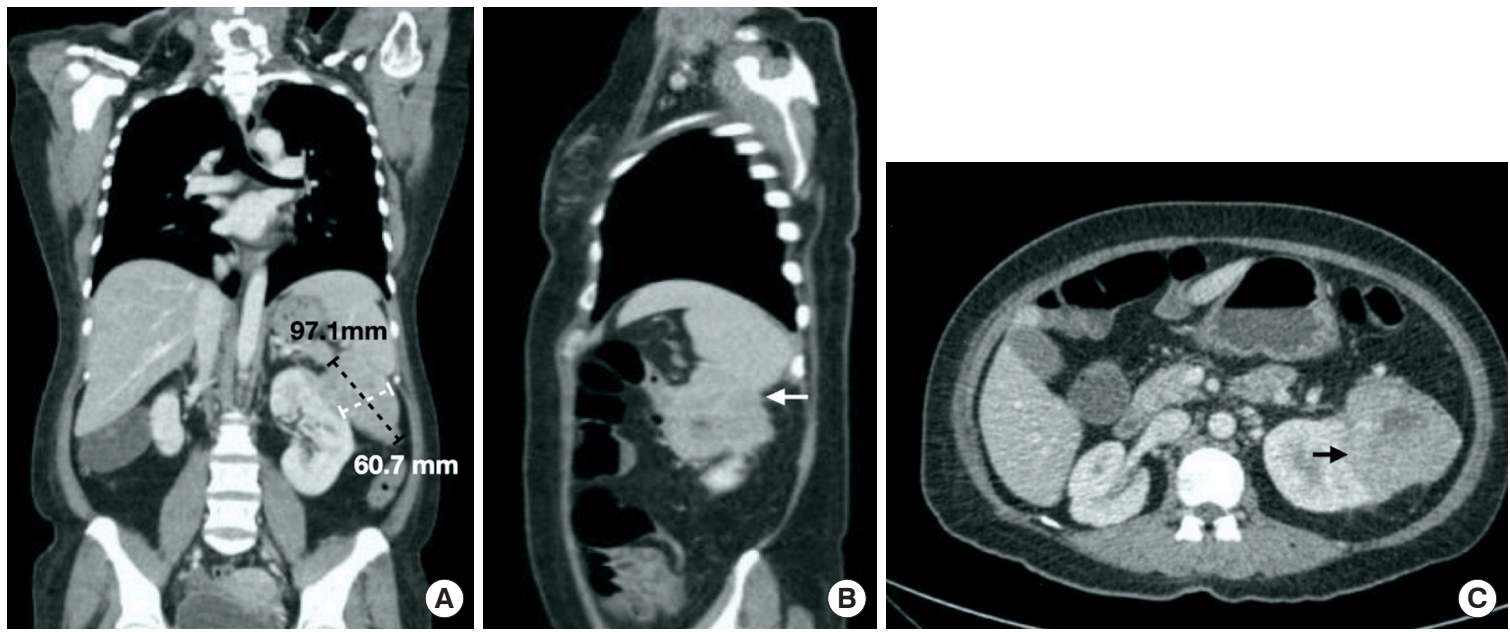

Fig. 2. Abdominal computed tomography scan. (A) Hyperdense mass with an infiltrative aspect measuring $9 \times 6 \mathrm{~cm}$ on the splenic angle of the colon. (B) Infiltration of the lower pole of the spleen (white arrow). (C) Concentric thickening of the entire colonic wall and secondary infiltration of the left kidney in the superior and anterosuperior segment (black arrow). 

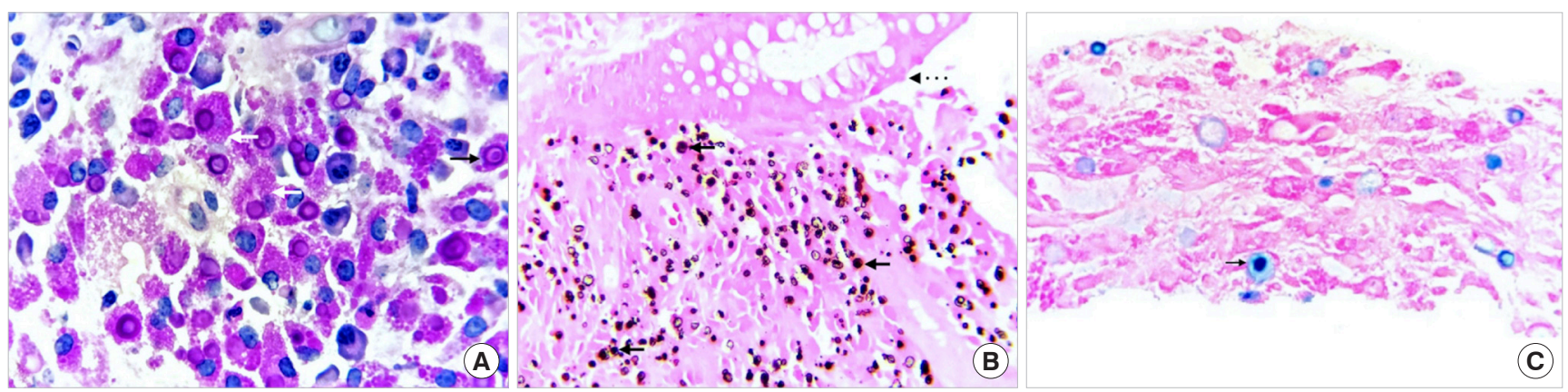

Fig. 3. (A) Photomicrograph of a histological section stained with periodic acid-Schiff (PAS), of the colonic tumor biopsy, which highlights a diffuse inflammatory infiltrate composed of histiocytes that present cytoplasms of granular appearance, von Hansemann cells (white arrows) with the presence of circular concentric calcifications, Michaelis-Gutmann bodies (black arrow) (both PAS positive, $\times 100$ ). (B) Photomicrograph of a histological section of the mucosa of the colon (upper portion) which presents a diffuse inflammatory infiltrate of the histiocytic type located in the lamina propria and submucosa (dotted arrow), the von Kossa stain (calcium) highlights the Michaelis-Gutman bodies (black arrows) $(\times 40)$. (C) Photomicrograph of a histological section of the colonic tumor biopsy, in which the Michaelis-Gutmann bodies are highlighted in blue, which contain iron and are positive for Perls staining (arrow) $(\times 100)$.

diagnosis of malakoplakia on the first specimen.

The patient was administered 500-mg oral ciprofloxacin every 12 hours for 12 weeks, and 2 weeks after completing the treatment, a restaging abdominal CT was performed, detecting a $20 \%$ increase in the volume of the colonic mass, and similar findings were observed in the spleen and kidney. A urologist was consulted, and the patient was scheduled to undergo a laparoscopic minimally invasive en bloc resection.

During surgery, a well-delimitated mass was observed, mainly involving the splenic flexure of the colon. A partial colectomy was performed through the infra-mesenteric approach. The mass severely adhered to the inferior border of the pancreatic tail, and the inferior splenic pole was difficult to mobilize. Significant bleeding was observed secondary to a partial lesion in the inferior splenic vessels. The proximal and distal colon were transacted with an endostapler and lateral-to-lateral stapled colonic anastomosis was established with a $60-\mathrm{mm}$ linear stapler. The mass was still attached to the superior pole of the kidney. The patient was positioned in the $30^{\circ}$ modified lateral decubitus position, and the urology team performed a partial nephrectomy with 2-layered nephrorraphy, a drain was left in the resection bed, and the procedure was completed without any other complications. The specimen was extracted through a Pfannenstiel incision (Fig. 4A).

Four days after the surgery, the patient presented with pain, fever, and chills. Common causes of postoperative sepsis were eliminated; based on the change in the color of the drained fluid; a sample was obtained, and the amylase level was reported to be $2,170 \mathrm{U} / \mathrm{L}$. Abdominal CT revealed an $11 \times 8 \times 8 \mathrm{~cm}$ area of peripancreatic fluid collection; hence, a pancreatic fistula was diagnosed. High-spectrum antibiotics, total parenteral nutrition, somatostatin, and percutaneous drainage were necessary for successful treatment of the fistula, and no further complications were detected. Oral feeding was restarted 10 days after the pancreatic
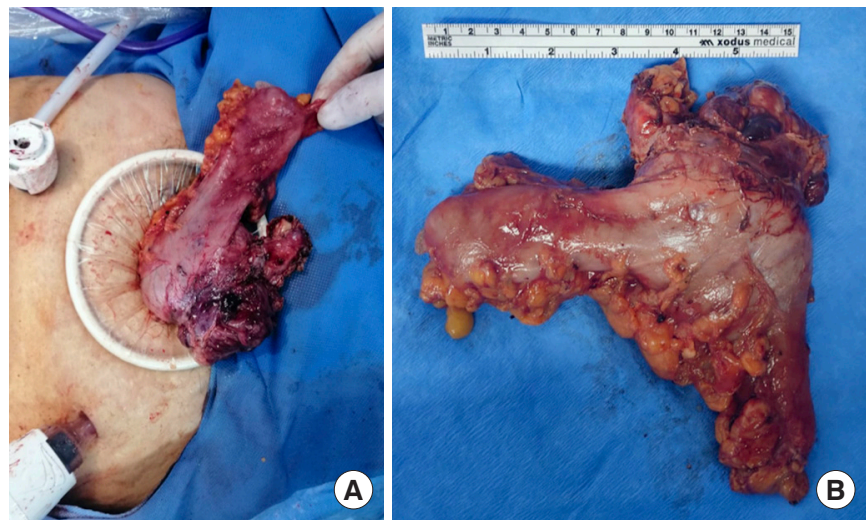

Fig. 4. (A) Surgical specimen extraction through a Pfannenstiel incision. (B) En bloc surgical specimen (including the splenic flexure and superior pole of the left kidney).

fistula treatment, and the patient was discharged 18 days after surgery. Histopathological examination confirmed the diagnosis of malakoplakia. Thirty-day follow-up was uneventful.

\section{DISCUSSION}

The urinary tract is involved in $75 \%$ of malakoplakia cases. When malakoplakia has a gastrointestinal origin, the clinical manifestations vary depending on the location, and the clinical spectrum may include acute abdomen, bleeding, diarrhea, urgency, perianal pain, painful evacuation, and even fistulas [3]. It is practically impossible to differentiate them radiologically and endoscopically from a neoplastic lesion or other digestive tract inflammatory diseases such as tuberculosis. In the gastrointestinal tract, the sigmoid colon and rectum are the most affected sites $[4,5]$. 
Diagnostic studies such as CT, magnetic resonance imaging, and endoscopy help diagnose the disease, determine the extent, and perform biopsies; however, the diagnosis is centered on the histopathological findings $[6,7]$. Multiple histiocytes with intracellular and extracellular Michaelis-Gutmann inclusion bodies are pathognomonic landmarks of this disease [2]. Although the precise etiology and pathogenesis of malakoplakia are unclear, it seems to originate from an acquired defect in the intracellular destruction capacity of phagocytosed bacteria due to an alteration in the degranulation of lysosomes [8]. The granulomatous reaction of malakoplakia results in the endoscopic appearance of the lesions, which vary from plaques to yellowish-brown nodules and masses. In the initial stages, malakoplakia commonly presents as yellowish mucous plaques, and as the disease progresses it presents as gray-brown polypoid lesions of varying sizes with or without peripheral erythema. Due to this endoscopic morphology, malakoplakia in the colon can be misdiagnosed as lymphoma, polyps, or carcinomas [9]. Although malakoplakia cases have been reported in immunocompetent patients, immunosuppression seems to be the most important predisposing factor for the development of this disease. In this case, the immunosuppressive condition was type $2 \mathrm{DM}$ with poor glycemic control and insulin dependence [3].

First-line treatment comprises empiric antibiotic therapy directed at the most frequently isolated microorganisms, with drugs that have a high concentration at the macrophage level, mainly quinolones, trimethoprim-sulfamethoxazole, and rifampicin [4]. Quinolones are the first-line treatment, with reported success rates of up to $90 \%$. Bethanechol chloride, a cholinergic agonist that increases cyclic guanosine levels and increases the bactericidal activity of damaged lysosomes, is also useful $[4,10]$. When there is a reversible cause of immunosuppression (corticosteroids, poorly controlled chronic diseases), treatment includes controlling or removing the cause for the same. Finally, surgical management is reserved for patients in whom medical treatment is ineffective or insufficient [4].

The association between malakoplakia and colon adenocarcinoma is common and has been previously reported. There are approximately 30 case reports in the English literature of patients in whom the tissue findings were compatible with malakoplakia has been identified as along with adenocarcinoma in the same specimen, and it is a cause of over-staging $[11,12]$. The association with other infections, such as cytomegalovirus and amebiasis has also been described [5].

Our case represents the challenges in diagnosing this pathological entity. The necessity of 2 biopsies for establishing an accurate diagnosis, the failure of antibiotic treatment, and a complex multidisciplinary surgical approach poses a challenge for the optimal management of this rare disease. Given the rarity of this pathology, high-quality evidence is lacking to guide optimal medical/ surgical treatment options.

\section{CONFLICTS OF INTEREST}

No potential conflict of interest relevant to this article was reported.

\section{ACKNOWLEDGMENTS}

We would like to thank Francisco E. Almazan U. M.D for his accurate endoscopic differential diagnosis and precise biopsies.

\section{REFERENCES}

1. Hyun KH, Shin HD, Kim DH. Malakoplakia in a healthy young female patient. Korean J Intern Med 2013;28:475-80.

2. Yousef GM, Naghibi B, Hamodat MM. Malakoplakia outside the urinary tract. Arch Pathol Lab Med 2007;131:297-300.

3. Bouguila J, Brahim K, Mokni M, Skandrani K, Harbi A, Essoussi AS, et al. Digestive malacoplakia in children: case report. ISRN Gastroenterol 2011;2011:597350.

4. Mitchell A, Dugas A. Malakoplakia of the colon following renal transplantation in a 73 year old woman: report of a case presenting as intestinal perforation. Diagn Pathol 2019;14:22.

5. Lee M, Ko HM, Rubino A, Lee H, Gill R, Lagana SM. Malakoplakia of the gastrointestinal tract: clinicopathologic analysis of 23 cases. Diagn Pathol 2020;15:97.

6. Pillay K, Chetty R. Malakoplakia in association with colorectal carcinoma: a series of four cases. Pathology 2002;34:332-5.

7. Park JW, Baek DH, Lee SJ. Multiple polypoid lesions in the sigmoid colon. Gastroenterology 2020;158:482-4.

8. Kohl SK, Hans CP. Cutaneous malakoplakia. Arch Pathol Lab Med 2008;132:113-7.

9. Thorlacius H, Jerkeman A, Marginean FE, Toth E. Colorectal malakoplakia in a patient with hypogammaglobulinemia. Gastrointest Endosc 2018;88:563-5.

10. Cipolletta L, Bianco MA, Fumo F, Orabona P, Piccinino F. Malacoplakia of the colon. Gastrointest Endosc 1995;41:255-8.

11. Niknejad N, Nili F, Shirkhoda M. Malakoplakia in association with adenocarcinoma of sigmoid colon: a case report. Iran J Pathol 2019;14:258-60.

12. Karasavvidou F, Potamianos SP, Barbanis S, Stathakis E, Psychos A, Kapsoritakis AN, et al. Malakoplakia of the colon associated with colonic adenocarcinoma diagnosed in colonic biopsies. World J Gastroenterol 2007;13:6109-11. 